\title{
A Narrative Review of the Impact of Aerobic Training on the Prevention and Treatment of Obesity-Related Hypertension
}

ISSN: 2576-8875

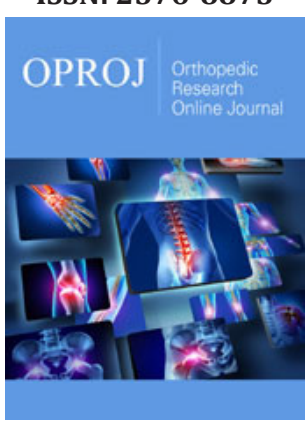

*Corresponding author: Onur Oral, Faculty of Sports Sciences, Department of Health Sciences and Sports, Ege University, Izmir, Turkey

Submission: 睯July 14, 2021

Published: 酸July 29, 2021

Volume 8 - Issue 3

How to cite this article: Onur Oral, Pınar Tatlibal, Evangelia Stavropoulou. A Narrative Review of the Impact of Aerobic Training on the Prevention and Treatment of Obesity-Related Hypertension. Ortho Res Online J. 8(3). OPROJ. 000689. 2021. DOI: 10.31031/OPROJ.2021.08.000689

Copyright@: Onur Oral, This article is distributed under the terms of the Creative Commons Attribution 4.0 International License, which permits unrestricted use and redistribution provided that the original author and source are credited.

\author{
Onur Oral ${ }^{1 *}$, Pinar Tatlibal ${ }^{2}$ and Evangelia Stavropoulou ${ }^{3}$ \\ ${ }^{1}$ Faculty of Sports Sciences, Ege University, Izmir, Turkey \\ ${ }^{2}$ Faculty of Sports Sciences, Dokuz Eylul University, Izmir, Turkey \\ ${ }^{3}$ Master of Research in Information and Communication, University of Lorraine, France
}

\begin{abstract}
Introduction: Obesity related hypertension has been appearing as a challenging health problem for years all over the world. For this reason, it has been underlined. That medical treatment methods should be combined with physical exercises. Recent studies show that aerobic exercises such as cycling, jogging assist weight loss in obese patients, which will lead the success of the medical treatment of hypertension.

Material and methods: In the search for scientific literature related to this review the US National Library of Medicine (PubMed) used MEDLINE and Sport discuss data and the terms 'aerobic exercise', 'hypertension', 'physical exercise' and 'obesity' were used. The relevant literature has also taken its source from the research of relevant articles from reference lists derived from data studies.

Result: Physical exercise, especially aerobic exercise has a significant role both in the treatment and prevention periods of obesity-related hypertension. In this sense, since aerobic exercises have been reported to provide benefits, it becomes a much more effective method in the treatment of hypertension, when the medical treatments of hypertension patients are combined with aerobic exercise programs.

Discussion: The regular exercise habits should also be taken into account as an effective method in the treatment of hypertension by taking care of the intensity and duration of the exercise to be applied should be considered in exercise planning.
\end{abstract}

Keywords: Aerobic exercise; Hypertension; Obesity

\section{Introduction}

The pressure that blood makes on the blood vessel wall is known as blood pressure (tension) and when blood pressure is higher than normal, this is called hypertension. In hypertensive individuals, risk factors which pertain to a great deal of systemic diseases, especially cardiovascular diseases, are significantly at high levels when compared to those who are healthy. Arterioles have the capacity of venoconstriction or vasodilation when they're stimulated by some neural and chemical factors. When the diameter of arterioles enlarges, blood can easily move and heart can pump blood without difficulty, on the other hand, when arterioles become narrow, the movement of blood grows difficult. In this way, in the event that the heart needs to pump blood with high and strong pressure, hypertension develops [1]. For blood pressure values that are measured in rest position, normal blood pressure is accepted as under $120 / 80 \mathrm{mmHg}$. Blood pressure values that are over $120 / 80 \mathrm{mmHg}$ (normal blood pressure), are defined as High Tension (Prehypertension, Phase 1 Hypertension, Phase 2 Hypertension); the follow-up and treatment of it should be regulated [2].

Those who have the values of 120-139/80-89mmHg which are defined as prehypertension, may be able to reduce their tension values under $120 \mathrm{mHg} / 80 \mathrm{mmHg}$ before they develop hypertension if they practice an appropriate exercise program, change their lifestyles like diet and lose weight. Therefore, only lifestyle change is recommended for those who participate in 
Prehypertension risk groups, while in addition to lifestyle change, drug therapy is recommended for those with Phase 1 Hypertension or higher risk cases. The risk of developing hypertension at later ages is $\% 90$ for a person who has normal blood pressure values and does not change his/her lifestyle [2].

Hypertension is a problem that threatens the health of people and society and if it's not controlled by proper treatment methods, it may lead to many fatal diseases like heart attack, stroke and renal impairment [3]. On the other hand, there's a close relationship between blood pressure and cardiovascular diseases and the risk of developing diseases like heart attack, coronary failure, stroke, eye and kidney diseases will increase as long as tension values go up [4-6].

On the other hand, it's stated that the adult population has 20\%-25\% hypertension prevalence in more developed industrial societies. It's also thought in developed countries that only approximately half of them is diagnosed. In the USA, \%27.4, in Germany \%22.5 of those who are diagnosed have received sufficient treatments [7]. The rate of hypertension patients whose blood pressures are under control is \%19 in Canada, \%6 in the United Kingdom [8]. Among the factors leading to hypertension, there are at a very high rate (over \%95) human metabolic structure including genetic factors and stress induced by environmental conditions, obesity caused by poor nutrition and sedentary lifestyle unprovided with physical activity. Kidney, vascular or hormonal diseases are responsible for the rest part (\%3-5) [9].

It's known that exercise has a protective effect against hypertension in treatment and control. Exercise programs containing especially aerobic activities for adults with normal and hypertensive blood pressure have protective effects against the development of hypertension [10]. Besides exercise's positive contributions to hypertension treatment, it's also determined that it can reduce the dose of drug used in hypertension treatment or eliminate the need of antihypertensive drugs [11]. Likewise, studies show that exercise reduces the risk of developing heart diseases and other chronic diseases, helps prevent obesity, consolidates muscle, bone and joints, and improves the quality of life. In order that the remedial effect of exercise in hypertension is at top-end, its severity, type, frequency and duration are essential. Literally exercise programs that should be run by hypertensive individuals should include continuous and rhythmic aerobic exercises concerning large muscle groups. These aerobic exercises are recommended as preferably at least between 3 and 5 days per week, moderate (\%40-\%60 V02max) and continuous or intermittent for 30-60min $[12,13]$.

Aerobic exercises are preferred as physical activity for the treatments of patients with hypertension [14,15]. Apart from increasing oxygenation of cardiac muscle, aerobic activities also help reaching ideal weight, reducing cholesterol level, controlling blood pressure and these metabolic developments also contribute to decrease psychological stress level, as a result, provides positive improvements in hypertension treatment [16].

\section{Discussion}

In a study carried out by Lee et al. [16] with 27 randomized control trials consisting of 1842 participants, an exercise program of walking for a mean of 19 week, which is between 4 days and 26 weeks, as an average of $36.5 \mathrm{~min} /$ day was practiced and a significant reduction of systolic blood pressure (5.2 to $11.0 \mathrm{mmHg}$ ) and diastolic blood pressure (3.8 to $7.7 \mathrm{mmHg}$ ) was observed [17].

Cornelissen and Smart also conducted a study with 105 trials including 3957 participants and a program of moderate aerobic exercise consisting of walking and jogging 3-5 times per week and 30-60 minutes per session for 4 and 52 weeks was carried out. As a result, a reduction in systolic blood pressure of $3.5 \mathrm{mmHg}$ and in diastolic blood pressure of $2.5 \mathrm{mmHg}$ was found out [18].

In addition, in a study carried out by Fagard et al. [18] consisting of 72 trials with an average of 40 participants per trial, 40 minutes sessions 3 times a week for 16 weeks at an average intensity of $65 \%$ of HR reserve, which is the difference between a person's maximum HR and resting HR, it was found out that a reduction in systolic BP of $6.9 \mathrm{mmHg}$ and in diastolic BP of $4.9 \mathrm{mmHg}$ had been observed [19].

Besides, in a study carried out by Cornelissen with 15 trials containing 633 participants, who practiced an exercise for 6-52 weeks, 30-60 minutes per session, 2-5 times per week at 50\%$70 \%$ HR reserve, it was found out that during daytime, a reduction in systolic BP of $3.2 \mathrm{mmHg}$ and in diastolic BP of $2.7 \mathrm{mmHg}$ was observed.

\section{Conclusion}

A large number of studies have been carried out to prevent hypertension, which has been increasing in recent years, or to find appropriate treatments, and numerous new treatment methods have been introduced. Since the positive effects of exercise have been proven many times in different studies, the positive effects of exercise in the prevention and treatment of hypertension cannot be ignored. In this sense, it is clear that especially aerobic exercises can provide important results for hypertension patients. Therefore, when medical treatments of hypertension patients are combined with exercise, it becomes a much more effective and powerful method in the treatment of hypertension, as indicated. For this reason, the effect of regular exercise habits should also be taken into account, and various factors such as the intensity and duration of the exercise to be applied should be considered in exercise planning.

\section{References}

1. Carpenter, Charles CJ (2003) Cecil essentials of medicine. Boston University, USA.

2. Chobanian AV, Bakris GL, Black HR, Cushman WC, Green LA, et al. (2003) Seventh report of the joint national committee on prevention, detection, evaluation, and treatment of high blood pressure. Hypertension 42(6): 1206-1252.

3. Dannenberg AL, Garrison RJ, Kannel WB (1998) Incidence of hypertension in the framingham study. Am J Public Health 78(6): 676679. 
4. Donnelly JE, Jacobsen DJ, Heelan KS, Seip R, Smith S (2000) The effects of 18 months of intermittent vs continuous exercise on aerobic capacity, body weight and composition, and metabolic fitness in previously sedentary, moderately obese females. International Journal of Obesity 24(5): 566-572.

5. Rêgo MLM, Cabral DA, Costa EC, Fontes EB (2019) Physical exercise for individuals with hypertension: It is time to emphasize its benefits on the brain and cognition. Clinical Medicine Insights: Cardiology 13: 1179546819839411.

6. WHO (2002) The world health report 2002-reducing risks, promoting healthy life. World Health Organization, Geneva, Switzerland, pp: 1-230.

7. WHO (2013) A global brief on hypertension: Silent killer, global public health crisis.

8. Moser M, Hebert PR (1996) Prevention of disease progression, left ventricular hypertrophy and congestive heart failure in hypertension treatment trials. J Am Coll Cardiol 27(5): 1214-1218.

9. Paolo RG, Taddei S, Virdis A, Ghiadoni L, Albertin G, et al. (2001) Exclusion of the ace I/D gene polymorphism as a determination of endothelial dysfunction. Hypertansion 37(2): 293-300.

10. Larsen MK, Matchkov VV (2016) Hypertension and physical exercise: The role of oxidative stress. Medicina 52(1): 19-27.

11. Mohan V, Seedat YK, Pradeepa R (2013) The rising burden of diabetes and hypertension in southeast asian and african regions: Need for effective strategies for prevention and control in primary health care settings. International Journal of Hypertension.
12. Leggio M, Fusco A, Limongelli G, Sgorbini L (2018) Exercise training in patients with pulmonary and systemic hypertension: A unique therapy for two different diseases. European Journal of İternal Medicine 47: 1724.

13. Rossi A, Dikareva A, Bacon SL, Daskalopoulou SS (2012) The impact of physical activity on mortality in patients with high blood pressure: A systematic review. Journal of Hypertension 30(7): 1277-1288.

14. Wallace JP (2003) Exercise in hypertension. Sports Medicine 33(8): 585598.

15. Daniel RM, Stelian S, Dragomir C (2010) The effect of acute physical exercise on the antioxidant status of the skeletal and cardiac muscle in the wistar rat. Romanian Biotechnol Lett 15(3): 56-61.

16. Lee LL, Watson MC, Mulvaney CA, Tsai CC, Lo SF (2010) The effect of walking intervention on blood pressure control: A systematic review. Int J Nurs Stud 47(12): 1545-1561.

17. Cornelissen VA, Smart NA (2013) Exercise training for blood pressure: A systematic review and meta-analysis. J Am Heart Assoc 2(1): e004473.

18. Fagard RH, Cornelissen VA (2007) Effect of exercise on blood pressure control in hypertensive patients. Eur J Cardiovasc Prev Rehabil 14(1): 12-17.

19. Cornelissen VA, Buys R, Smart NA (2013) Endurance exercise beneficially affects ambulatory blood pressure: A systematic review and metaanalysis. J Hypertens 31(4): 639-648.

For possible submissions Click below: 